ADMISSION BLOOD GLUCOSE

\title{
Admission blood glucose as a prognostic indicator in patients with acute myocardial infarction
}

Raisul I. Russel", Naresh Ranjith ${ }^{\#}$ and Benn Sartorius ${ }^{\dagger}$

*Department of Medicine, Nelson R. Mandela School of Medicine, University of KwaZulu-Natal, Durban, South Africa

\#Cardiac and Heart Failure Centre, City Hospital (JMH), Durban, South Africa

Department of Public Health Medicine, School of Nursing and Public Health, University of KwaZulu-Natal, Durban, South Africa

Address for correspondence:

Prof N. Ranjith

Cardiac and Heart Failure Centre

City Hospital (Joint Medical Holdings)

83 Ismail C Meer Street

Durban

4001

South Africa

Email:

ranjith@lantic.co.za

\section{INTRODUCTION}

Coronary heart disease (CHD) is a major cause of morbidity and mortality. ${ }^{(1)}$ This is particularly important in people with diabetes because they are at increased risk of having CHD, and to have multi-vessel disease when it occurs. ${ }^{(2)}$ Patients with both diabetes and acute myocardial infarction (AMI) are more likely to present with acute pulmonary oedema, and this increased incidence of heart failure occurs despite similar infarct sizes and left ventricular ejection fractions. ${ }^{(3,4)}$ This observation suggests that the left ventricle in diabetes tolerates infarction poorly, irrespective of whether patients present with ST elevation myocardial infarction (STEMI) or non-ST elevation myocardial infarction (NSTEMI).

Another important consideration in patients with $\mathrm{AMI}$ is the positive association between the admission glucose values and mortality in patients with and without diabetes. ${ }^{(5)}$ There appears to be a graded relationship between admission glucose levels with major adverse cardiac events (MACE) both during hospital admission and post discharge. ${ }^{(6)}$ Although several mechanisms have been proposed as to why there are worse outcomes in patients presenting with $\mathrm{AMI}$ and higher glucose concentrations on admission, it is still unclear whether high blood glucose

\section{ABSTRACT}

Objective: To investigate the utility of admission blood glucose for predicting major adverse cardiac events (MACE) during hospitalisation and 6 months' postdischarge in acute myocardial infarction (AMI) patients. Methods and results: This study recruited 2878 AMI patients admitted to the Coronary Care Unit at R. K. Khan Hospital, Durban, South Africa, from 2002 - 2014. Demographic and clinical data stored in an electronic database were obtained from all patients. Admission blood glucose levels were sub-divided into 3 groups; low (<7.8), medium (7.8-10.9) and high ( $\geq \mathrm{II}) \mathrm{mmol} / \mathrm{l}$. The mean age of the study population was $57.18 \pm 7$ years of whom $65 \%$ were males. Self-reported diabetes was

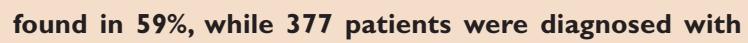
diabetes based on HbAlc levels $\geq 6.5 \%$, increasing the overall prevalence to $72 \%(n=2070)$. More patients were in the low admission blood glucose group (49\%), medium group (16\%), and high group (35\%). The highest prevalence of MACE was seen in the high group (42\%) compared to either the medium (39\%) or low groups (26\%; $\mathrm{p}<0.001$ ), particularly for cardiogenic shock $(p<0.00 I)$, cardiac failure $(p<0.00 I)$ and death $(p<0.00 I)$. Following multivariable logistic regression analyses of clinical and laboratory parameters associated with mortality, high admission blood glucose conferred a significantly increased odds of mortality $(p=0.001)$. The optimal cut-off admission blood glucose value as determined via the receiver operating characteristic curve for predicting in hospital and 6 months' mortality was $8.5 \mathrm{mmol} / \mathrm{l}$ (AUC of 0.63 ) and $8.1 \mathrm{mmol} / \mathrm{l}$ (AUC of $0.6 \mathrm{l}$ ) for MACE.

Conclusions: This study shows that patients have multiple risk factors for AMI with diabetes playing a central role. Although elevated admission blood glucose is an important predictor for in hospital and shortterm MACE, the cut-off value for predicting MACE and mortality has only modest predictability and further research is required to improve the performance of these measures for routine clinical use.

SAHeart 2019;16:1 18-126

is a marker for a sicker patient, as opposed to being causative for worse outcomes.

The optimal blood glucose cut-off value for the prediction of poor outcomes in AMI patients has not been determined. 
Recommendations for glycaemic control comes from 3 population groups, namely: critically ill patients from intensive care units, patients admitted with AMI, and general medical wards, ${ }^{(7,8)}$ all of which have their limitations. This may explain why the optimal strategy for the management of hyperglycaemia in patients with and without diabetes admitted with AMI is largely unknown. However, there is general consensus around the following points, hypoglycaemia should be avoided, and blood glucose be maintained between 7 - $10 \mathrm{mmol} / \mathrm{I}^{(9)}$

In South Africa, the prevalence of CHD is high, especially amongst the Asian Indian population, who present with multiple risk factors for $\mathrm{AMI}$, which probably explains why they present at an early age. ${ }^{(10)}$ More importantly, in addition to other conventional risk factors, self-reported diabetes was found in $46 \%$ of these patients with AMI. Considering the limited information available on glycaemic control on prognosis after an $\mathrm{AMI}$, the present study therefore was undertaken to assess the utility of admission blood glucose for predicting MACE, both in hospital and 6 months' post-discharge in AMI patients with and without diabetes. Furthermore, we also sought to determine the optimal cut-off admission blood glucose level for predicting poor outcomes in these patients.

\section{METHOD}

\section{Study population}

In this retrospective, observational study, we enrolled patients admitted to the Coronary Care Unit (CCU) at R. K. Khan Hospital, Durban, South Africa, with a diagnosis of AMI. The study was carried out according to the principles of the Declaration of Helsinki and approved by the local ethics committee. From 2002 - 20।4, 3 I 42 patients were admitted to the CCU with a diagnosis of acute coronary syndrome, but only a total of 2878 patients who fulfilled the inclusion criteria of AMI, and who had admission blood glucose results available, were enrolled into the study. Acute myocardial infarction was defined by prolonged chest discomfort, typical electrocardiographic changes, and elevated cardiac troponin T (cTnT) levels, as outlined by the Joint European Society of Cardiology/ American College of Cardiology Committee. (II)

\section{Clinical assessment}

Demographic and clinical data stored in an electronic database were obtained from all patients and analysed. This data were collected in real time during patient's admission to the CCU and all information was reviewed and validated by one of the co-authors. These included: age, gender, anthropometric measurements (body mass index and abdominal waist circumference), and risk factors for CHD including a family history of vascular disease, clinical findings on admission, types of $\mathrm{AMI}$, echocardiographic changes, treatment during hospitalisation and after discharge. A detailed description of complications encountered during hospital admission and 6 months' post discharge such as ventricular arrhythmias, complete heart block, heart failure, cardiogenic shock, cerebrovascular accident (CVA), recurrence of angina/infarction, and death were recorded.

In keeping with standard acute coronary syndrome guidelines, all patients were counselled and offered coronary angiograms, which were performed at another referral hospital. Patients were required to consent for both coronary angiography and cardiac revascularisation procedures such as percutaneous coronary intervention ( $\mathrm{PCl}$ ) and coronary artery bypass grafting ( $C A B G$ ), should there be a need to perform these procedures. This type of triage is necessary due to the large volume of work and the lack of adequate facilities in the public sector (only I government-based hospital in the province of KwaZulu-Natal offers these specialised facilities). Consequently, only a limited number of patients underwent coronary angiography because the majority declined cardiac revascularisation. Patients who were eligible were thrombolysed with metalyse, as a revascularisation therapy.

\section{Biochemical analyses}

Blood samples were obtained intravenously from all subjects in the CCU immediately after hospital admission to quantify serum blood glucose concentrations and other biochemical measurements. Analyses were carried out using standard methods.

Plasma glucose levels were measured using the Dimension EXL (Siemens Healthineers). Furthermore, we sub-divided admission blood glucose levels into 3 groups, low ( $<7.8 \mathrm{mmol} / \mathrm{l})$, medium $(7.8-10.9 \mathrm{mmol} / \mathrm{l})$ and high $(\geq 1 \mathrm{lmmol} / \mathrm{l})$, to assess whether an increasing degree of admission blood glucose levels was associated with an increased risk of MACE. The cut-off values for the 3 different categories of admission blood glucose was closely based on the 2-hour glucose values of the oral glucose tolerance test because the cut-off values for admission blood glucose, which discriminates between good and poor prognosis, varies over a wide range. Total cholesterol, triglycerides (TGs), high-density lipoprotein cholesterol (HDL-C), and low-density lipoprotein cholesterol (LDL-C) were measured 48 hours after admission following a $9-12$ hour over- 
night fast. Troponin T was measured on the Elecsys 2010 (Roche Diagnostics) and the test was considered positive at a cut-off value $>0.03 \mathrm{ng} / \mathrm{ml}$.

\section{Statistical analyses}

Data were analysed using Stata 13.I and R. Mean with standard deviation (SD) was used for continuous variables, or median with interquartile range (IQR) was used instead if the variable was skewed. Categorical data were presented using frequency tables. Association between categorical variables was assessed using the standard Pearson's chi-square $(\times 2)$ test. If expected cell count in the cross tabulation had fewer than 5 observations (sparse numbers) then the Fisher's exact test was applied. The standard student's t-test was used to compare the mean of continuous explanatory variables by dichotomous outcome classification. If the normality assumption was not upheld, then the non-parametric equivalent, namely the Wilcoxon rank-sum test, was used instead. Factors associated with mortality were assessed using bivariate and multivariable logistic regressions. This was performed to include the confounding influence of other variables (e.g. age, gender, etc.) in the multivariable adjusted estimates. Coefficients were exponentiated to present odds ratios (ORs) and 95\% confidence intervals (Cls). Significance in the adjusted models was assessed at $p<0.05$. Model fit (adequacy) was assessed using goodness of fit tests. The Youden index was used to identify the optimal breakpoints in blood glucose concentration in terms of its predictive capability for mortality and MACE. The discriminatory power was assessed using the AUC. An AUC $\geq 0.8$ suggests good to excellent predictive capability. Sensitivity, specificity, positive predictive value, and negative predictive values based on the identified optimal cut-points were calculated. A p-value of $<0.05$ was taken as statistically significant.

\section{RESULTS}

The study cohort comprised 2878 patients with AMI, of whom 2269 (79\%) presented with STEMI (Table I). The mean age of the study population was $57.18 \pm 11.47$ years with a male preponderance (65\%). Data were missing for certain variables such as abdominal girth ( $n=440,15 \%)$ and HbAlc $(n=574$, 20\%). Smoking (61\%), self-reported diabetes (59\%), hypertension (53\%), previous myocardial infarction (12\%) and previous angina (12\%) were the most commonly observed risk factors. Fifty-eight percent of the patients had a family history of coronary artery disease, diabetes (43\%), hypertension (38\%) and cerebrovascular disease (19\%). Of the $41 \%$ of patients who did not present with a self-reported history of diabetes, HbAIc
TABLE I: Baseline characteristics of patients.

\begin{tabular}{|c|c|}
\hline Characteristics & $\begin{array}{c}\text { Summary } \\
\mathrm{n}=2878(\%)\end{array}$ \\
\hline Male & | 874 (65) \\
\hline ST elevation myocardial infarction & $2269(79)$ \\
\hline Non-ST elevation myocardial infarction & $609(21)$ \\
\hline \multicolumn{2}{|l|}{ Body mass index } \\
\hline Normal $\left(\leq 25 \mathrm{~kg} / \mathrm{m}^{2}\right)$ & | 249 (44) \\
\hline Overweight $\left(26-29 \mathrm{~kg} / \mathrm{m}^{2}\right)$ & I 040 (36) \\
\hline Obese $\left(\geq 30 \mathrm{~kg} / \mathrm{m}^{2}\right)$ & $589(20)$ \\
\hline \multicolumn{2}{|l|}{ Risk factors } \\
\hline Smoking & $|75|(61)$ \\
\hline Self-reported diabetes & | 693 (59) \\
\hline Newly diagnosed diabetes ( $\mathrm{HbA} \mid \mathrm{C} \geq 6.5 \%)$ & $377(13)$ \\
\hline Total diabetic population & $2070(72)$ \\
\hline With nephropathy (eGFR $<60 \mathrm{~mL} / \mathrm{min} / 1.73 \mathrm{~m}^{2}$ ) & $586(28)$ \\
\hline With retinopathy & $363(18)$ \\
\hline Hypertension & $1516(53)$ \\
\hline Previous myocardial infarction & $359(12)$ \\
\hline Previous angina & $34 \mid(12)$ \\
\hline \multicolumn{2}{|l|}{ Family history of vascular disease } \\
\hline Coronary artery disease & I $657(58)$ \\
\hline Diabetes & | 238 (43) \\
\hline Hypertension & I 104 (38) \\
\hline Cerebrovascular disease & $543(19)$ \\
\hline \multicolumn{2}{|l|}{ Medication at discharge } \\
\hline ACEIs/ARBs & $2295(80)$ \\
\hline Beta blockers & I 770 (62) \\
\hline Antiplatelets & $2862(99)$ \\
\hline Nitrates & 2847 (99) \\
\hline Statins & $2738(95)$ \\
\hline Calcium antagonists & $200(9)$ \\
\hline \multicolumn{2}{|l|}{ Diabetic treatment } \\
\hline Oral hypoglycaemic drugs & I $169(56)$ \\
\hline Insulin & $516(25)$ \\
\hline Insulin + oral hypoglycaemic drugs & $385(19)$ \\
\hline \multicolumn{2}{|l|}{ Continuous variables } \\
\hline Age (years) mean (SD) & $57.18 \pm 11.47$ \\
\hline \multicolumn{2}{|l|}{ Median (IQR) } \\
\hline Abdominal girth $(\mathrm{cm})(\mathrm{n}=2438)$ & $97(90-105)$ \\
\hline Female & $99(92-107)$ \\
\hline Male & $96(89-104)$ \\
\hline Heart rate (bpm) & $82(70-96)$ \\
\hline Systolic BP (mmHg) & $130(|||-| 50)$ \\
\hline Diastolic BP (mmHg) & $80(70-91)$ \\
\hline \multicolumn{2}{|l|}{ Biochemical data } \\
\hline Hemoglobin $(g / d l)$ & $14(12.5-15.3)$ \\
\hline Admission blood glucose $(\mathrm{mmol} / \mathrm{l})$ & $7.8(5.8-13.1)$ \\
\hline $\mathrm{HbAIc}(\%)(n=2304)$ & $6.9(6-9)$ \\
\hline Creatinine $(\mu \mathrm{mol} / \mathrm{l})$ & $92(79-112)$ \\
\hline Troponin T (ng/ml) & $2.14(0.58-5.27)$ \\
\hline Total cholesterol (mmol/l) & $5.2(4.3-6.02)$ \\
\hline LDL cholesterol $(\mathrm{mmol} / \mathrm{l})$ & $3.3(2.59-4.02)$ \\
\hline HDL cholesterol (mmol/l) & $0.94(0.79-1.13)$ \\
\hline Triglycerides $(\mathrm{mmol} / \mathrm{l})$ & $1.67(1.17-2.43)$ \\
\hline
\end{tabular}

$I Q R=$ Interquartile Range; Oral hypoglycemic drugs included Metformin, Sulphonylureas, or combination of both. 
TABLE II: Baseline characteristics \& outcomes of patients according to admission blood glucose.

\begin{tabular}{|c|c|c|c|c|}
\hline \multirow[b]{2}{*}{ Characteristics } & \multicolumn{4}{|c|}{ Blood glucose } \\
\hline & 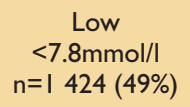 & $\begin{array}{c}\text { Medium } \\
7.8-10.9 \mathrm{mmol} / / \mathrm{l} \\
\mathrm{n}=456(16 \%)\end{array}$ & $\begin{array}{c}\text { High } \\
\geq 1 \mathrm{Immol} / \mathrm{l} \\
\mathrm{n}=998 \text { (35\%) }\end{array}$ & $\mathrm{p}$-value \\
\hline Age (years) Mean (SD) & $55.46( \pm 11.73)$ & $59.91( \pm 11.07)$ & $58.39( \pm 10.89)$ & $<0.00$ । \\
\hline \multicolumn{5}{|l|}{ Gender } \\
\hline Female & $327(23)$ & $196(43)$ & $481(48)$ & \multirow{2}{*}{$<0.001$} \\
\hline Male & । 097 (77) & $260(57)$ & $517(52)$ & \\
\hline Non-ST elevation myocardial infarction & $293(21)$ & $106(23)$ & $210(21)$ & \multirow{2}{*}{0.475} \\
\hline ST elevation myocardial infarction & I I3| (79) & $350(77)$ & $788(79)$ & \\
\hline Pharmacological reperfusion ( $n=87 \mid$ ) & $440(40)$ & $127(36)$ & $304(39)$ & 0.673 \\
\hline \multicolumn{5}{|l|}{ Body mass index } \\
\hline Overweight vs. normal BMI & 491 (42) & $169(48)$ & $380(49)$ & 0.003 \\
\hline Obese vs. non-obese & $254(18)$ & $108(24)$ & $227(23)$ & 0.002 \\
\hline \multicolumn{5}{|l|}{ Risk factors } \\
\hline Smoking & I 024 (72) & $248(54)$ & $479(48)$ & $<0.001$ \\
\hline Hypertension & $579(4 I)$ & $303(66)$ & $634(64)$ & $<0.001$ \\
\hline Diabetes & $390(27)$ & $348(76)$ & $955(96)$ & $<0.001$ \\
\hline Previous angina & $147(10)$ & $82(18)$ & $112(11)$ & $<0.001$ \\
\hline Previous myocardial infarction & $166(12)$ & $70(15)$ & $123(12)$ & 0.114 \\
\hline \multicolumn{5}{|l|}{ Family history of vascular disease } \\
\hline Hypertension & $497(35)$ & $172(38)$ & $435(44)$ & $<0.001$ \\
\hline Diabetes & $47 \mid(33)$ & $224(49)$ & $543(54)$ & $<0.001$ \\
\hline Coronary artery disease & $802(56)$ & $284(62)$ & $57 \mid(57)$ & 0.078 \\
\hline Cerebrovascular disease & $275(19)$ & $88(19)$ & $180(18)$ & 0.708 \\
\hline \multicolumn{5}{|l|}{ Killip class } \\
\hline । & | 327 (96) & $396(93)$ & $814(91)$ & Ref \\
\hline 2 & $54(4)$ & $29(7)$ & $81(9)$ & $<0.001$ \\
\hline 3 & $34(2)$ & $19(4)$ & $63(7)$ & $<0.001$ \\
\hline 4 & $7(0.5)$ & II (3) & $35(4)$ & $<0.001$ \\
\hline Major adverse cardiovascular events & $370(26)$ & $175(39)$ & $415(42)$ & $<0.001$ \\
\hline Cardiac failure & $142(10)$ & $92(20)$ & $231(23)$ & $<0.001$ \\
\hline Death & $80(6)$ & $49(11)$ & $136(14)$ & $<0.001$ \\
\hline Cardiogenic shock & $31(2)$ & $32(7)$ & $81(8)$ & $<0.001$ \\
\hline Recurrence of infarct & $96(7)$ & $39(9)$ & $62(6)$ & 0.255 \\
\hline Recurrence of angina & $57(4)$ & $25(6)$ & $46(5)$ & 0.392 \\
\hline Atrial fibrillation & $34(2)$ & $15(3)$ & $23(2)$ & 0.498 \\
\hline Supraventricular tachycardia & II (0.8) & $5(1)$ & $8(0.8)$ & 0.795 \\
\hline Ventricular arrhythmia & $48(3)$ & $19(4)$ & $44(4)$ & 0.397 \\
\hline Complete heart block & $25(2)$ & $5(1)$ & $36(4)$ & 0.002 \\
\hline Cerebrovascular accidents & $18(1)$ & $9(2)$ & $23(2)$ & 0.142 \\
\hline Coronary angiogram & $n=580$ & $n=180$ & $n=339$ & \\
\hline Single vessel disease & 151 (26) & $34(19)$ & $72(21)$ & 0.160 \\
\hline Double vessel disease & $165(29)$ & $55(31)$ & $91(27)$ & \\
\hline Triple vessel disease & $264(46)$ & $91(5 \mathrm{I})$ & $176(52)$ & \\
\hline Coronary artery bypass graft & $308(53)$ & $90(50)$ & $184(54)$ & 0.646 \\
\hline Percutaneous coronary intervention + stent & $131(23)$ & $45(25)$ & $73(22)$ & 0.667 \\
\hline Continuous variables & \multicolumn{4}{|c|}{ Median (IQR) } \\
\hline Heart rate (bpm) & $80(68-91)$ & $84.5(72-98)$ & $86(73-101)$ & $<0.001$ \\
\hline Systolic BP (mmHg) & $128(110-149)$ & $130(|1|-150)$ & $132(114-153)$ & 0.005 \\
\hline Diastolic BP (mmHg) & $80(70-91)$ & $79(70-90)$ & $80(70-91)$ & 0.877 \\
\hline Abdominal girth $(\mathrm{cm})(\mathrm{n}=2 \mathrm{438})$ & $95(88-102)$ & $99(92-107)$ & $99(92-106)$ & $<0.001$ \\
\hline Female & $97(89-105)$ & $101(93-108)$ & $100(93-107)$ & 0.006 \\
\hline Male & $95(87-102)$ & $98(92-107)$ & $98(91-106)$ & $<0.001$ \\
\hline Ejection fraction & $54(46-59)$ & $54(43-58)$ & $52(43-58)$ & $<0.001$ \\
\hline
\end{tabular}


TABLE II continued: Baseline characteristics \& outcomes of patients according to admission blood glucose.

\begin{tabular}{|c|c|c|c|c|}
\hline \multirow[b]{2}{*}{ Characteristics } & \multicolumn{4}{|c|}{ Blood glucose } \\
\hline & $\begin{array}{c}\text { Low } \\
<7.8 \mathrm{mmol} / \mathrm{I} \\
\mathrm{n}=\mathrm{I} 424(49 \%)\end{array}$ & $\begin{array}{c}\text { Medium } \\
7.8-10.9 \mathrm{mmol} / \mathrm{l} \\
\mathrm{n}=456(16 \%)\end{array}$ & $\begin{array}{c}\text { High } \\
\geq 1 \mathrm{Immol} / \mathrm{l} \\
\mathrm{n}=998(35 \%)\end{array}$ & $\mathrm{P}$-value \\
\hline \multicolumn{5}{|l|}{ Biochemical data } \\
\hline Hemoglobin (g/dl) & $14.2(13-15.4)$ & $13.6(12.1-15.1)$ & $13.6(12.1-15.3)$ & $<0.00$ । \\
\hline $\mathrm{HbAlc}(\%)(n=2304)$ & $6.3(5.8-7.8)$ & $7.4(6.2-9)$ & $8.2(6.4-10)$ & $<0.00$ । \\
\hline $\mathrm{HbAIc} \geq 6.5 \mathrm{n}(\mathrm{col} \%)$ & $510 / 1121(46)$ & $246 / 359(69)$ & $613 / 824(74)$ & $<0.00$ । \\
\hline Creatinine $(\mu \mathrm{mol} / \mathrm{L})$ & $92(80-108)$ & $94(78-114)$ & $93(76-117)$ & 0.810 \\
\hline Total cholesterol (mmol/l) & $5.16(4.4 I-5.96)$ & $5.27(4.42-6.07)$ & $5.23(4.44-6.1)$ & 0.142 \\
\hline LDL cholesterol $(\mathrm{mmol} / \mathrm{l})$ & $3.34(2.65-4.05)$ & $3.25(2.60-4.07)$ & $3.26(2.54-3.99)$ & 0.191 \\
\hline $\mathrm{HDL}$ cholesterol (mmol/l) & $0.95(0.79-1.14)$ & $0.93(0.80-1.12)$ & $0.94(0.8-1.14)$ & 0.661 \\
\hline Triglycerides (mmol/l) & $1.53(1.08-2.22)$ & $1.76(1.28-2.55)$ & $1.85(1.28-2.78)$ & $<0.00$ । \\
\hline Peak Troponin T (ng/ml) & $2.25(0.55-5.37)$ & $1.78(0.39-4.89)$ & $2.23(0.67-5.21)$ & 0.087 \\
\hline
\end{tabular}

IQR = Interquartile Range.

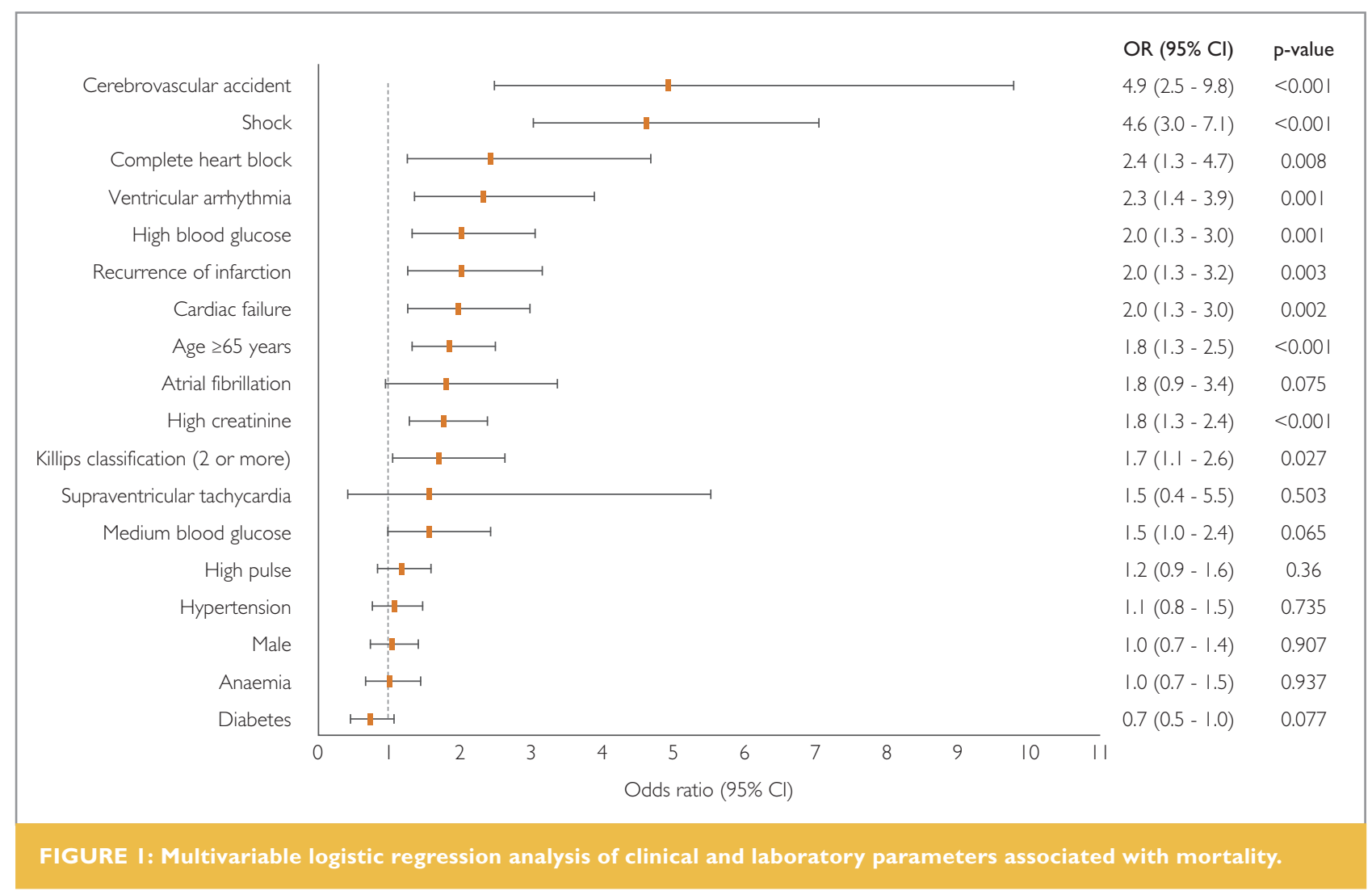

levels were performed on admission in 930 subjects. An additional $41 \%(n=377)$ were diagnosed with diabetes based on $\mathrm{HbAlc}$ levels $\geq 6.5 \%$, increasing the prevalence of diabetes in the study cohort to $72 \%(n=2070)$. Twenty-eight percent $(n=586)$ had diabetic nephropathy (estimated glomerular filtration rate of $\left.<60 \mathrm{~mL} / \mathrm{min} / 1.73 \mathrm{~m}^{2}\right)$, while $18 \%(\mathrm{n}=363)$ presented with diabetic retinopathy.
The median (interquartile range) admission blood glucose and $\mathrm{HbAIc}$ levels were 7.8 (5.8 - I3.I ) mmol/I and 6.9 (6.0\% - 9.0\%), respectively. The majority of patients were on optimal medical therapy at hospital discharge. All patients in hospital with hyperglycaemia were treated with an insulin-based regime to try to maintain blood glucose levels less than $10 \mathrm{mmol} / \mathrm{l}$ and hypoglycaemia was strictly avoided. Following discharge, 56\% 
were on oral hypoglycaemic agents $(\mathrm{OHA})$ that included either metformin, sulphonylureas or both, insulin alone (25\%), or a combination of insulin and $\mathrm{OHA}(19 \%)$.

Baseline characteristics and clinical outcomes of study participants according to admission blood glucose categories are summarised in Table II. The majority of patients were in the low
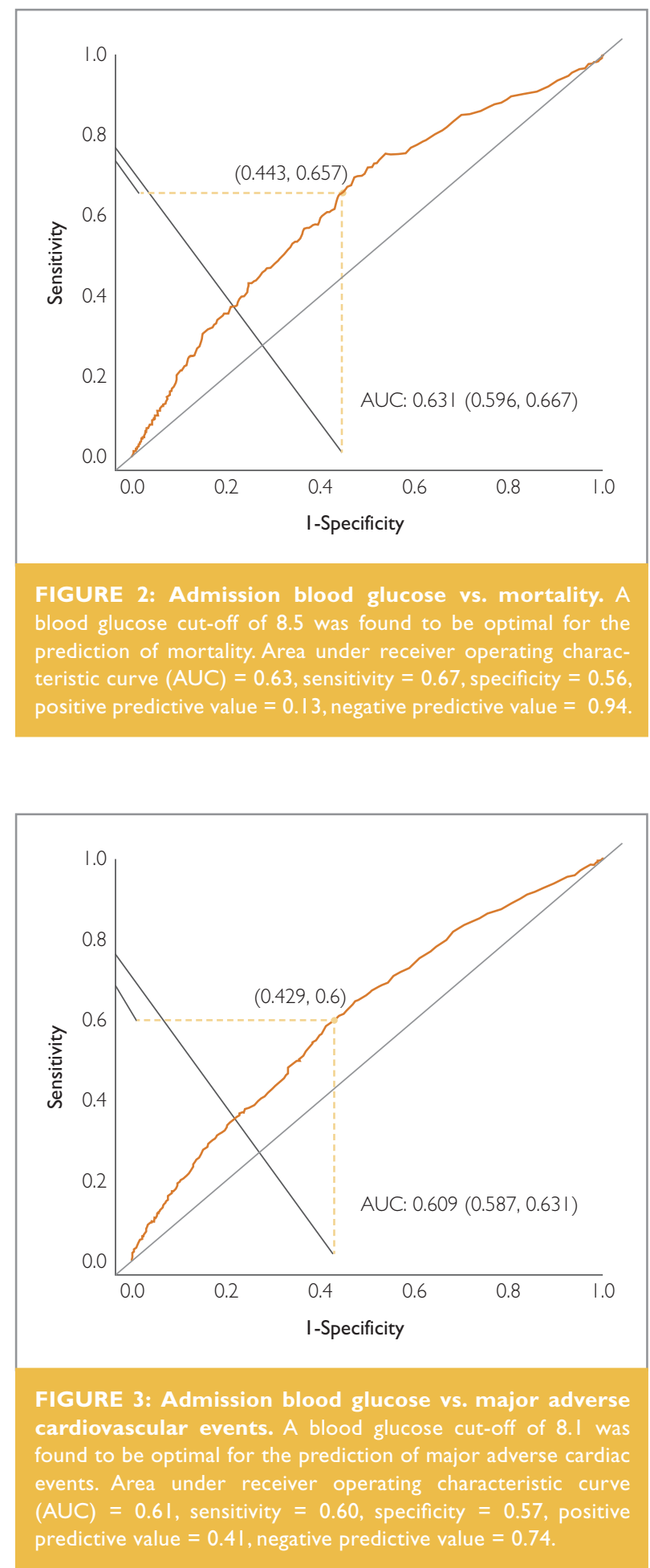

admission blood glucose group (49\%), while 16\% presented in the medium admission blood glucose group and $35 \%$ in the high admission blood glucose group. A significantly greater number of male patients presented in the low admission blood glucose group $(77 \%, p<0.001)$ when compared to their female counterparts who were more likely to have higher admission blood glucose values $(p<0.00 I)$. Obese patients as assessed by $\mathrm{BMI}$ and those with increased visceral obesity as determined by abdominal waist circumference measurements were also found to have significantly higher admission blood glucose levels $(p=0.002$ and $p<0.00 \mathrm{I}$, respectively). As expected, diabetic patients had higher admission blood glucose levels (high group $96 \%$, medium group $76 \%$ compared to the low group $27 \%$, $\mathrm{p}<0.00 \mathrm{I})$. A similar trend was observed for patients with hypertension $(p<0.00 \mathrm{I})$ and for those with a family history of diabetes $(p<0.00 \mathrm{I})$ and hypertension $(p<0.00 \mathrm{I})$. Patients with high admission blood glucose levels had lower ejection fraction on echocardiography $(p<0.00 \mathrm{I})$, abnormal Killip class $(p<0.00 \mathrm{I})$, decreased median serum haemoglobin ( $13.6 \mathrm{~g} / \mathrm{dL}$, [IQR 12.1 I5.3], $\mathrm{p}<0.00 \mathrm{I})$, hypertriglyceridaemia $(1.85 \mathrm{mmol} / \mathrm{I}$, [IQR 1.28 2.78], $p<0.00 \mathrm{I}$ ) and higher HbA Ic levels (8.2\%, [IQR 6.4 - I0], $\mathrm{p}<0.00$ I).

Analysis of MACE showed that the highest prevalence of MACE was observed in the high admission blood glucose group (42\%) when compared to either the medium group (39\%) or the low admission blood glucose group (26\%), $p<0.00$ I. This was particularly evident for cardiogenic shock $(p<0.00$ I), cardiac failure $(p<0.00 \mathrm{I})$ and death $(p<0.00 \mathrm{I})$. No significant correlation was seen between the 3 study groups for angiographic severity of disease and coronary revascularisation. Similarly, no significant association was found for the $38 \%$ $(n=87$ I) of STEMI patients who received thrombolysis.

A summary of the multivariable analysis for identifying factors associated with mortality by logistic regression analysis is shown in Figure I. In addition to cerebrovascular accidents $(p<0.00$ I), cardiogenic shock $(p<0.0 \mathrm{l})$, complete heart block $(p=0.008)$, ventricular arrhythmias $(p=0,00 \mathrm{I})$, recurrence of infarction $(p=0.003)$, cardiac failure $(p=0.002)$, age $\geq 65$ years $(p<0.00 \mathrm{I})$, high creatinine $(p<0.00 \mathrm{I})$ and Killip class $\geq 2(p=0.027)$, patients with high admission blood glucose levels also conferred a significant increased odds of mortality $(p=0.00 \mathrm{I})$.

We plotted the AUC with identified optimal cut-off value of admission blood glucose for predicting mortality in patients with AMI (Figure 2). These analyses suggested that optimal blood glucose cut-off identified in this cohort (admission blood 
glucose of $8.5 \mathrm{mmol} / \mathrm{l}$ ) had modest predictive capability for mortality with an AUC of 0.63 and corresponding sensitivity and specificity of 0.67 and 0.56 , respectively. Similarly, the optimal blood glucose cut-off identified in this study for MACE (admission blood glucose of $8.1 \mathrm{mmol} / \mathrm{l}$ ) also displayed modest predictive capability with an AUC of 0.61, and corresponding sensitivity of 0.60 and specificity 0.57 (Figure 3 ).

\section{DISCUSSION}

Individuals with diabetes mellitus are at increased risk for the development of $\mathrm{AMI}^{(12,13)}$ and diabetes is now considered a coronary risk equivalent by the National Cholesterol Education Programme.(14) The current study confirms these findings with the prevalence of self-reported diabetes being present in 59\% of patients with AMI. An additional 377 patients were diagnosed with diabetes based on $\mathrm{HbAlc}$ levels, suggesting that the more accurate prevalence of diabetes in the study cohort was $72 \%$. Given the widespread use of $\mathrm{HbAlc}$ in the diagnosis and management of patients with diabetes, we believe that the use of $\mathrm{HbAlc}$ in the majority of patients is a key strength of our study. The presence of elevated levels of $\mathrm{HbAlc}$ levels in these patients makes stress hyperglycaemia a less likely diagnosis, indicating that the metabolic abnormality preceded the infarction. These results concur with a recent local study which compared different diagnostic criteria for the detection of undiagnosed diabetes in 896 acute myocardial infarction patients (data unpublished). Of interest is that using $\mathrm{HbAIc}$ criteria, $32 \%$ of patients were diagnosed with diabetes while $47 \%$ were diagnosed with pre-diabetes, highlighting the increased prevalence of diabetes in this geographical region. Our findings support the importance of screening for glucose abnormalities in patients hospitalised for AMI to strengthen secondary preventative efforts (including medical and lifestyle interventions) in a high-risk patient population.

Patients in the study also presented with multiple conventional risk factors such as smoking, hypertension, dyslipidemia, obesity, and a family history of vascular disorders, which may also possibly have contributed to the occurrence of AMI. Therefore, a comprehensive risk factor intervention strategy is required over and above strict glycaemic control to decrease the risk of complications following an AMI.

Several studies have reported a positive association between higher glucose concentrations, adverse clinical outcomes and mortality in patients with AMI both during hospital admission and post-discharge. ${ }^{(15,16)}$ Although the mechanisms by which hyperglycaemia cause harmful effects in patients with AMI are unclear, it has been suggested that a wide variety of pathophysiological changes are involved. These include volume depletion, increased free fatty acid production, decreased nitric oxide availability, increased coagulopathy and atherogenesis, endothelial dysfunction and increased circulating inflammatory cytokines. ${ }^{(17-19)}$ Our findings are in agreement with previous reports. Thirty-five percent of patients presented in the high admission blood glucose group and this group experienced a significantly greater number of MACE when compared to either the medium or low admission blood glucose groups $(p<0.00 \mathrm{l})$. Of note, is that a significantly greater proportion of diabetic patients and those with the highest $\mathrm{HbAlc}$ values were found in the high admission blood glucose group, indicating that they had poor glycaemic control prior to their infarction. Patients in the high admission blood glucose group were significantly more likely to be hypertensive, obese, and have a worse Killip class.

Cardiac failure, cardiogenic shock and death were the most common adverse events observed in the high admission blood glucose group when compared to the lower admission blood glucose groups. These results concur with an analysis of 15 different trials that addressed the prognostic significance of admission blood glucose concentrations in patients with AMI. ${ }^{(20)}$ The pathogenesis of heart failure in diabetes is multifactorial, but can be largely attributable to ischaemic heart disease, hypertension, diabetic cardiomyopathy and extracellular fluid volume expansion. ${ }^{(21,22)}$ More importantly, cardiac metabolism is altered in the presence of heart failure due to decreased mitochondrial function as a result of the accumulation of medium and long chain acyl-carnitine, increased ketone metabolism and the accumulation of lactate and branch chain amino acids. ${ }^{(23)}$ Patients with diabetes, therefore, are at increased risk for the development of heart failure and the prevention of heart failure is becoming a major emerging research and treatment goal such as the discovery of the sodium-glucose co-transporter 2 (SGLT2) inhibitors for the treatment of diabetes and coexistent heart failure. ${ }^{(24)}$ Thus, our findings provide a contemporary picture in a South African cohort of patients suggesting that elevated admission blood glucose levels are a prognostic marker for in-hospital and short-term adverse clinical outcomes in patients with AMI.

Furthermore, following multivariable logistic regression analyses of clinical and laboratory parameters associated with mortality, in addition to cerebrovascular accidents, cardiogenic shock, complete heart block, ventricular arrhythmias, recurrence of 
infarction, cardiac failure age $\geq 65$ years, high creatinine and Killip class $\geq 2$, high admission blood glucose also conferred a significant increased odds of mortality.

In addition to the admission value, a graded relationship has been found between increasing blood glucose levels and MACE. However, the optimal glucose cut-off value for the prediction of poor outcomes has not been determined. We also investigated the optimal cut-off admission blood glucose value as determined via a ROC curve for predicting in-hospital and 6 months' mortality in patients with AMI. The optimal cut-off point was an admission blood glucose value of $8.5 \mathrm{mmol} / /$, which has only modest capability for predicting mortality. Similarly, the optimal cut-off point for MACE was an admission blood glucose value of $8.1 \mathrm{mmol} / /$, which also displayed modest predictive capability. Hence, these cut-off values are only exploratory and further studies are required before they may serve as indicators of clinical reference for secondary prevention for improving the prognosis after AMI. We are unaware of any large studies in South Africa that examined the prevalence and predictive value of admission blood glucose in patients presenting with $\mathrm{AMI}$ and our study may provide important epidemiological insight into elevated admission blood glucose as a risk factor for MACE. Although $\mathrm{HbAlc}$, which reflects metabolic and glycaemic control, has been shown to be a significant predictor of MACE after AMI, ${ }^{(25)}$ elevated admission blood glucose may serve as a cost-effective and easily obtainable alternate prognostic marker.

\section{LIMITATIONS}

Several potential limitations need acknowledgement. First, this was a retrospective observational study with few variables having missing data, which may have caused selection bias. This shortcoming was minimised by using standardised methods for data collection, while the large study population allowed for greater statistical power. Second, the follow-up period of the patients was only 6 months; thus, it is necessary to investigate the longer-term relationship between admission blood glucose and MACE. Third, although we reveal that elevated admission blood glucose was associated with unfavorable in-hospital and 6-month outcome, the effects of treating hyperglycaemia after admission were not assessed. Finally, 62\% of patients with STEMI did not receive thrombolytic therapy because of late presentation or standard contraindications, while only $38 \%$ of patients underwent coronary angiographic studies, with no patients receiving primary $\mathrm{PCl}$ according to timeline-based guidelines. Therefore, these data should be interpreted with caution, particularly with respect to the various MACE outcomes observed. This is due to the fact that patients are referred to another hospital with long waiting lists for cardiac catheterisation, and a large number of them declined intervention or did not have coronary angiograms performed because of other co-morbidities.

\section{CONCLUSIONS}

The findings from the present study show that patients have multiple risk factors for AMI with diabetes playing a central role. Elevated admission blood glucose in AMI patients is an important indicator for in-hospital and short-term MACE, with a graded increase in the risk of MACE demonstrated with increasing levels of admission blood glucose. Although the optimal blood glucose target in AMl patients with hyperglycaemia is uncertain, the cut-off admission blood glucose value for predicting MACE and mortality in our study has only modest predictability and further research is required to improve the performance of these measures for routine clinical use.

\section{Conflict of interest: none declared.}




\section{REFERENCES}

I. Mendis S, Puska P, Norrving B (201 I). World Health Organisation. Global atlas on cardiovascular disease prevention and control [online]. http://www.who.int/cardiovascular_diseases/publications/atlas_cvd/en/

2. Haffer SM, Lehto S, Ronnemaa T, et al. Mortality from coronary heart disease in subjects with type 2 diabetes and in non-diabetic subjects with and without prior myocardial infarction. N Engl J Med 1998;339:229-34.

3. Granger CB, Califf RM, Young S, et al. Outcome of patients with diabetes mellitus and acute myocardial infarction treated with thrombolytic agents. I Am Coll Cardiol 1993;21:920-25.

4. Stone PH, Muller JE, Hartwell $\mathrm{T}$, et al. The effect of diabetes mellitus on prognosis and serial left ventricular function after acute myocardial infarction: Contribution of both coronary disease and diastolic left ventricular dysfunction to the adverse prognosis. J Am Coll Cardiol 1989; 14:49-57.

5. Kosiborod M, Rathore SS, Inzucchi SE, et al. Admission glucose and mortality in elderly patients hospitalised with acute myocardial infarction: Implication for patients with and without recognised diabetes. Circulation 2005; I | |:3078-86.

6. Suleiman M, Hammerman $\mathrm{H}$, Boulos $\mathrm{M}$, et al. Fasting glucose is an important independent risk factor for 30-day mortality in patients with acute myocardial infarction: A prospective study. Circulation 2005; I I 1:754-60.

7. Kosiborod M, Inzucchi SE, Goyal A, et al. Relationship between spontaneous and iatrogenic hypoglycaemia and mortality in patients hospitalised with acute myocardial infarction. JAMA 2009;301:1556-64.

8. Cheung NW, Wong WW, McLean M. The Hyperglycaemia: Intensive Insulin Infusion in Infarction (HI-5) study: A randomised controlled trial of insulin therapy for myocardial infarction. Diabetes Care 2006;29:765-70.

9. Finney SJ, Zekveld C, Elia A, et al. Glucose control and mortality in critically ill patients. JAMA 2003;290:204 I-47.

10. Ranjith N, Pegoraro RJ, Zaahl MG. Risk factors associated with acute coronary syndromes in South African Asian Indian patients (The AIR Study). J Clinic Experiment Cardiol 201 1;2:163-68.

II. Alpert JS, Thygesen K, Antman E, et al. Myocardial infarction redefined: A consensus document of The Joint European Society of Cardiology/ American College of Cardiology Committee for the redefinition of myocardial infarction. J Am Coll Cardiol 2000;36:959-69.

12. Kannel WB, McGee DL. Diabetes and cardiovascular disease. The Framingham study. JAMA 1979;241:2035-38.

3. Yusuf S, Hawken S, Ounpuu S, et al. Effect of potentially modifiable risk factors associated with myocardial infarction in 52 countries (the INTERHEART study): Case-control study. Lancet 2004;364:937-52.

14. National Cholesterol Education Programme (NCEP) expert panel on detection, evaluation and treatment of high blood cholesterol in adults (Adult Treatment Panel III). Third report of the National Cholesterol Education Programme (NCEP) expert panel on detection, evaluation, and treatment of high blood cholesterol in adults (Adult Treatment Panel III) final report. Circulation 2002; 106:3143-421.

15. Pres D, Gasior M, Strojek K, et al. Blood glucose level on admission determines in-hospital and long-term mortality in patients with ST-segment elevation myocardial infarction complicated by cardiogenic shock treated with percutaneous coronary intervention. Kardiol Pol 201 0;68:743-5I.

16. Savonitto S, Morici N, Cavallini C. One-year mortality in elderly adults with non-ST-elevation acute coronary syndrome: Effect of diabetic status and admission hyperglycaemia. J Am Geriatr Soc 2014;62:1297-303.

17. Marfella R, Nappo F, De Angelis L, et al. Haemodynamic effects of acute hyperglycaemia in type 2 diabetic patients. Diabetes Care 2000;23:658-63.

8. Packer M. Activation and inhibition of sodium-hydrogen exchanger is a mechanism that links the pathophysiology and treatment of diabetes mellitus with that of heart failure. Circulation 2017; 1 36:1548-59.

9. Esposito K, Nappo F, Marfella R, et al. Inflammatory cytokines concentrations are acutely increased by hyperglycaemia in humans: Role of oxidative stress. Circulation 2002:106:2067-72.
20. Capes SE, Hunt D, Malmberg K, et al. Stress hyperglycaemia and increased risk of death after myocardial infarction in patients with and without diabetes: A systemic overview. Lancet 2000;355:773-78.

21. Nichols GA, Gullion CM, Koro CE, et al. The incidence of congestive heart failure in type 2 diabetes: An update. Diabetes Care 2004;27:1879-84.

22. Dei Cas A, Khan SS, Butler J, et al. Impact of diabetes on epidemiology, treatment, and outcomes of patients with heart failure. JACC Heart Fail 20I5;3:136-45

23. McGarrah RW, Crown SB, Zhang GF, et al. Cardiovascular Metabolomics. Circ Res 2018;122:1238-58.

24. Zinman B, Wanner C, LachinJM, et al. Empagliflozin, cardiovascular outcomes, and mortality in type 2 diabetes. N Engl J Med 2015;373:21 17-28.

25. Chen CL, Yen DH, Lin CS, et al. Glycated haemoglobin level is an independent predictor of major adverse cardiac events after non-fatal acute myocardial infarction in non-diabetic patients: A retrospective observational study. Medicine 2017;96:6743. 